\title{
Review
}

\section{Surgical treatment of acquired valvular heart disease in elderly patients}

Kubatbek S. Urmanbetov, Elmira N. Tukusheva, Tursunbek B. Kaliev, Sultanmurat Zhumabaev

Scientific Research Institute of Heart Surgery and Transplantation of Organs of the Ministry of Health of the Kyrgyz Republic, Bishkek, Kyrgyzstan

\begin{abstract}
This article provides an overview of studies on indications, surgery and outcomes of valvular heart disease surgery in elderly patients.
\end{abstract}

Key words: elderly, cardiovascular surgery, valvular heart disease surgery, coronary bypass surgery

(Heart Vessels and Transplantation 2018; 2: doi 10.24969/hvt.2017.48)

\section{Introduction}

The development of surgical techniques for valvular heart disease (VHD) began with the commissurotomy in rheumatic mitral stenosis with access from the left ventricle and / or the left atrium without the use of an artificial circulatory system in the early 1950s (1). With the advent of cardiopulmonary bypass, effective artificial valves and adequate intraoperative protection of the myocardium, surgical treatment of valvular defects became the method of choice $(2,3)$.

Surgical possibilities also expand with the improvement of valvular prostheses development (4, 5). Worldwide, a large number of different types of prostheses are used, which take into account the characteristic of damage to the valve apparatus. Reconstructive operations are becoming more common. The approaches to management of VHD differ also according to the underlying cause of valvular heart disease and presence of concomitant cardiovascular diseases that more often are encountered in elderly.

\section{VHD surgery results in elderly}

According to data of patients with mitral valve (MV) disease derived from database in North America in 1990s, MV replacement surgery (MVR) was performed in $28.2 \%$ of all heart valve replacement surgeries, among them indication was mitral stenosis
(MS) in $28.2 \%$, mitral insufficiency $-38.6 \%$ and mixed disease $-38.6 \%$ (6). In the presence of MS, surgical treatment often depends on the level of development of the health care system: in some countries (including Russia), endovascular balloon commissurotomy is practically not used, while in other countries this method is used in almost one third of patients (7).

In a European study (8), among patients with AV disease and indications for surgery underwent AV replacement surgery (AVR) was performed in $49 \%$ patients with aortic stenosis and $76.5 \%$ - with aortic regurgitation. Among cases with mitral insufficiency, the mitral valve repair was performed in $46.5 \%$ of patients, and the mechanical prosthesis was implanted on $43.2 \%$. In case of MS, a mechanical prosthesis was implanted in 58\% of cases and percutaneous balloon commissurotomy was used in $33.9 \%$ of cases. In addition, in $31.7 \%$ of patients one or more concomitant interventions were performed, mainly coronary bypass surgery (CABG) $(22.8 \%)(8)$. In southern Europe, mechanical prostheses were used in $70.4 \%$ of cases for AVR and in $83 \%$ for MVR. In Northern Europe - the numbers were, $60.9 \%$ and $83.4 \%$ respectively. However, there were no significant differences in the incidence of mitral valve use: $44.3 \%$ of patients with mitral regurgitation in the US (8), and $46.5 \%$ in European countries (9).

Address for Correspondence: Elmira Tukusheva, Scientific Research Institute of Heart Surgery and Organ Transplantation, Togolok Moldo 3/1, Bishkek, Kyrgyzstan, Email: etukusheva@mail.ru

Received: 12.02.2018 Accepted: 05.04.2018 
According to the research carried out in Russian center, among patients older than 65 years, AVR was performed in $42.9 \%$ of the patients, MVR in $31.2 \%$ of patients, multi-valve replacement in $11.6 \%$ of patients, and valve repair in $29.1 \%$ of patients. CABG was performed simultaneously with valvular surgery in $25.9 \%$ of cases (10). In another series from the same center, mechanical prosthesis was used in $58.9 \%$ of patients older than 60 years with MV disease, in $13.4 \%$ of cases, a biological prosthesis was implanted; valve repair was performed on $27.7 \%$ of patients. When aortic valve was damaged, mechanical prosthesis was used in $75.8 \%$ of cases, in $22.5 \%$ of patients a biological prosthesis was used while, reconstructive operations on the valve were performed in $2 \%$ of cases. In the correction of functional or organic tricuspid insufficiency, valve repair was performed in $90 \%$ of cases (11). According to Schelbert et al (12), among 87585 patients older than 65 years (with mean age of 76 years) operated in 1,045 US clinics for heart defects, only AVR constituted the most of surgeries - in 91.8\%. MVR simultaneously with AVR were performed in 5735 (6.5\%) patients, operation on tricuspid valve in 126 $(0.14 \%)$ patients and pulmonary artery valve in 20 (0.022\%) patients (12).

The methodology of managing patients evolves over time. In a study performed in three US clinics, Roberts et al. (13) compared the rate of different types of surgeries for 35 years and noted the reduction in the proportion of surgeries for $\mathrm{AV}$ disease associated with congenital anomalies (single-leaf and bicuspid valve) and increase in the proportion of simultaneous CABG and AVR (among men from 0 to $57 \%$, among women from 0 to 45\%) (13). In Norway, from 1979 to 2004, the rate of interventions associated with ischemic and degenerative mitral insufficiency also increased. Of all the interventions, $40 \%$ of patients underwent a standalone mitral valve surgery; $33 \%$ in combination with CABG and $13 \%$ in combination with intervention on the aortic valve. In other cases, there was a combination with tricuspid valve correction and other surgical interventions (14).

The choice of surgical treatment on acquired heart defects depends on the hemodynamic significance of the defect, the presence or absence of clinical symptoms, the presence of concomitant pathology, and age. Worldwide, there is no uniform criteria for determining the severity of complications and worse outcomes, more often the classification is based on the features of hemodynamics detected by ECG and heart catheterization and imaging, and examination of the data $(15,16)$. It is generally accepted that the testimony of patients should be individualized due to the fact that there still exist differences in the opinions of specialists in determining the indications for the operation, depending on the degree of hemodynamic significance of the defect, ejection fraction, age, concomitant pathology; there is a discrepancy between opinions on the optimal time, methodology and conditions for implementation.

\section{VHD surgery combined with CABG in elderly}

Often, in elderly patients, it becomes necessary to perform CABG simultaneously with valvular heart surgery. According to Roberts et al. (13), over the past three decades, the number of patients on whom, CABG and valve replacement were performed simultaneously has increased by $57 \%$ among men, and by $45 \%$ among women. According to Urmanbetov (11), surgical correction of valvular heart disease with CABG in the elderly was performed in $32.8 \%$ of cases, statistically significant differences were also found in the groups of patients aged; $60-65$ years $(24.2 \%), 65$ 70 years $(37.1 \%)$ and over 70 years $(42 \%)$ for the frequency of CABG concomitant with valve defect correction. The type of operation performed (implantation of mechanical, biological prosthesis or reconstructive surgery) is also closely related to the age group of patients (11).

In 2012, Dell'Amore, et al. (17) published the results of AVR with or without CABG in patients older than 80 years of age. Hospital mortality for all patients was $5.3 \%$ (in AVR group - $4.3 \%$ vs AVR plus CABG - $7.2 \%, p$ $=0.29$ ). Among patients who underwent $A V R$ in combination with CABG, the history of myocardial infarction was more often recorded, neurological deficit and multifocal atherosclerosis; in the intraoperative period, the time of aortic clamping was higher, and in the postoperative period, the low cardiac output syndrome was more often registered (early outcomes - $18.6 \%$ in group AVR+CABG, compared with $4.8 \%$ in group AVR). Differences in results, according to the authors, are due to the initial clinical characteristics of patients (17).

In Norway, during the same period, the proportion of patients with ischemic and degenerative mitral insufficiency in whom the CABG and MVR or mitral valve repair were simultaneously performed was $33 \%$ while, $13 \%$ of patients simultaneously underwent CABG and AVR (18). 
According to European recommendations dating back to 2012 (19), the CABG and AVR are indicated even in presence of a moderate degree of aortic stenosis (aortic valve area 1.0-1.5 $\mathrm{cm}^{2}$ ). AVR is also recommended in the presence of indications for CABG, if the age is 70 years, the peak pressure gradient exceeds $30 \mathrm{~mm} \mathrm{Hg}$ and there is a progression of aortic stenosis, expressed in an increase in the pressure aortic valve gradient by $5 \mathrm{~mm} \mathrm{Hg}$ per year. However, in each specific case, an individual solution of the multidisciplinary team is required, taking into account the body surface area, hemodynamic data, the degree of calcification, the rate of progression of aortic stenosis, life expectancy, the presence and extent of concomitant diseases, and the individual risk of the operation. The combined implementation of percutaneous coronary intervention and transcatheter aortic valve implantation (TAVI) is possible, but, according to the European recommendations, it is necessary to conduct methodological qualitative studies before the recommendations are formulated. The choice of operations should be the subject of individual discussion based on the patient's clinical condition, coronary anatomy and myocardium in the risk zone (19).

In addition to the combined operations, elderly patients experience conditions that are more rarely recorded among young patients. For example, a narrow left ventricular outflow tract and a narrow fibrotic aortic valve fibrous ring may require expansion of the fibrous ring, and severe calcification of the valve, ring, and aortic root may require complex procedure of prosthetic valve implantation and an intervention on ascending aorta. Preoperative preparation of elderly patients with severe left ventricular hypertrophy, accompanied by appropriate intraoperative treatment, can significantly reduce mortality and the incidence of complications.

Given that in elderly patients mitral malformation is often combined with chronic atrial fibrillation, it is necessary to resolve the issue of combined operations. The Maze procedure can prevent thromboembolism in the future by maintaining a normal sinus rhythm. The decision to perform the Maze procedure should be based on the patient's age and health, as well as on the surgical experience of the surgeon, since this procedure can increase the risk of surgery (20).

\section{Tricuspid valvular surgery in elderly}

In elderly patients, tricuspid valve dysfunction is also often recorded. The duration of surgery for a tricuspid valve failure, as well as the type of surgical intervention, has caused controversy. In recent years, annuloplasty has become a common surgical approach with a significant deficiency of the tricuspid valve, less frequent use of a low-profile mechanical valve or bioprosthesis. Because of the high risk of thromboembolic complications with the use of mechanical prostheses in the tricuspid position, the biological prosthesis is more preferable. In 2011, Topilsky et al. performed a retrospective analysis of the results of tricuspid valve replacement in severe tricuspid regurgitation in 189 patients (37\% of which were men, aged $67.5 \pm 11.3$ years). The operational mortality rate was $10 \%$; the risk of mortality was significantly higher if the use of intra-aortic balloon pump (IABP) was required $(\mathrm{OR}=3.2,95 \% \mathrm{Cl} 1.9-5.6 \%$, $\mathrm{p}<0.0001$ ); with severe heart failure (NYHA IV compared with class II or III, OR $=1.7 ; 95 \% \mathrm{Cl} 1.05$ $2.8 \% ; p=0.02)$. Over the follow-up period $(29.3 \pm 27.1$ months), mortality was $37 \%$. In $3 \%$ of cases, a reoperation was required and $21.7 \%$ of patients experience recurrence of heart failure symptoms. The absence of cardiovascular events (death, re-operation on the tricuspid valve, heart failure) was noted in $41.3 \%$ of patients (21).

\section{Surgery of VHD and carotid artery disease}

Often among elderly patients, a combination of valvular malformation and stenosis of carotid arteries is recorded, so the choice of methodology for managing patients with such pathology is of particular importance (22).

In 2004, Yoda et al. published results of treatment of 79 patients who underwent a one-stage operation of carotid endarterectomy (CE) and prosthetic heart valves implantation $(63.2 \%$ of men, mean age $-68.9 \pm 6.9$ years). Indication for the $\mathrm{CE}$ was the narrowing of the lumen of the carotid artery by more than $75 \%$, the intervention on the valvular apparatus of the heart - the presence of a hemodynamically significant valve defect. AVR was performed in 64 patients, MVR - 10, replacement of aortic and mitral valves - 5 patients. Operational mortality was $10.1 \%$ (8 patients), in the same series of patients, the early postoperative period was complicated by acute stroke in two patients. 
It was revealed that double-valve replacement with prosthetic valves is an independent risk factor for early operational mortality $(p=0.039)$, and myocardial infarction and age over 70 years were risk factors for early postoperative mortality $(p=0.022$ and $p=0.03$ respectively). For the development of acute cerebrovascular events, both in the early and late postoperative period, there were no statistically significant independent risk factors. The authors believe that the operation of CE in combination with AVR could be considered an operation in which the benefit is greater than the risk, the accumulation of experience is necessary for other types of operations (23). The same group of authors, in 2011, published the results of another small observational study with 15 patients (mean age of $68.9 \pm 6.7$ years) on whom one-stage operations were performed: $\mathrm{CE}$ in combination with CABG (14 patients) or valve correction (1 patient). The main indication for $\mathrm{CE}$ was $75 \%$ stenosis of the carotid artery in combination or without ischemic cerebral lesions (stroke or transient ischemic attack), in the anamnesis it was registered in 8 patients. There were no lethal outcomes, cerebral circulation disorder in the postoperative period was revealed in 1 patient (6.7\%) (24).

Several studies analyzed outcomes of different timing of interventions in patients with combined pathologies $(25,26)$. In 2010, Kar et al. (25) published the results of aortic stenosis treatment (AV area $<1.0 \mathrm{~cm}^{2}$ ) among patients with carotid artery stenosis in Cleveland Clinic, USA from 1998 to 2005. The incidence of severe aortic stenosis among this group of patients was $6.3 \%$. As first stage of treatment, 28 patients $(54 \%)$ underwent stenting of the carotid arteries. Overall, $10 \%$ of patients (of whom $6 \%$ died within 30 days after stenting) did not survive the second stage of treatment (AVR). At the same time, in the group of patients in whom only AVR was performed, there were no lethal cases or acute disturbance of cerebral circulation during the 1- year of follow-up. Birchley et al. (26) presented the results of treatment of patients with carotid disease and VHD: 100 patients underwent CE before open-heart surgery (in a screening study of 11394 patients, critical carotid stenosis was revealed in $100-0.87 \%$ ); then, CABG was performed in 80 patients, the rest underwent CABG in combination with VHD surgery. Stroke developed in $1 \%$ of patients after carotid endarterectomy and before CABG, and in $2 \%$ after CABG. Mortality after CE was $2 \%$. The combined end-point (redo-CABG, myocardial infarction and / or death) was $7.5 \%$ after CABG and $10.2 \%$ after all operations. The performance of step-by- step operations led to a decrease in the absolute risk of perioperative acute cerebrovascular event- by $0.3 \%$ compared with the operation without CE and 30-day mortality rate was $1.4 \%(26)$. According to Anselmi et al. (27), among patients who underwent surgical correction of valvular defects, asymptomatic carotid artery stenosis (>50\%) was detected in $26.4 \%$ and stenosis $>70 \%$ - in $3.6 \%$ of patients. The methodology of management was chosen as follows: for asymptomatic stenosis less than $69 \%$ - isolated correction of valvular defect, with stenosis of $70 \%$ or more - a combined operation (correction of the defect $+\mathrm{CE}$ ). According to the authors, the mortality and frequency of complications did not differ statistically (27).

Currently, there are no randomized studies, could clearly define the methodology of management of such patients. According to the consensus reached, bilateral carotid artery disease ( $\geq 70 \%$ of stenosis) should be considered clinically significant in assessing the risk of stroke in the short term period after cardiac surgery (28). However, carotid carcinoma itself may not be a factor, but a marker of the risk of such complications, since hemodynamically significant lesions of carotid arteries often involve lesions of the aorta and intracerebral vessels, which leads to acute impairment of cerebral circulation in the early postoperative period. That is why it is necessary to conduct large-scale research.

\section{Conclusion}

In general, the considerable variability of the types of operations performed in elderly patients with VHD, as well as concomitant cardiac pathology in elderly patients, hampers the creation of statistically homogeneous groups and limits the ability to perform comparative studies to assess the clinical efficacy of surgical procedures. In clinical terms, such variability of operations becomes the basis for making medical decisions based on the experience and expert opinions of specialists, the impossibility of creating clear recommendations based on evidence.

Peer-review: internal and external

Conflict of interest: None to declare

Authorship: K.S.U., E.N.T., T.B.K., S.Z. equally contributed to the preparation of manuscript Acknowledgement and funding: None to declare 


\section{References}

1.National guidelines for the management, diagnosis and treatment of valvular heart disease. Bakulev Scientific Center of Cardiovascular Surgery RAMS, Moscow 2009; p356.

2.Marcinkevicius AM, Palyushchinskaya NA, Kibarskite $\mathrm{N}$. Long-term results of mitral valve prosthesis depending on prosthesis model. Thorac Cardiovasc Surg 1981; 5: 35-8.

3.Skopin II. Multicomponent reconstructive surgery on the mitral valve. M., 1992. (dissertation Doctor of Medical Sciences)

4.Karaskov AM, Zheleznev SI, Nazarov VM. Use of mechanical artificial heart valves "Medlnzh-2" with a modified braid for surgical correction of acquired heart diseases. Bulletin Bakulev Scientific Center of Cardiovascular Surgery RAMS, 2009; 2: 108-14.

5.Karaskov AM, Semenov II , Astapov DA. Implantation of epoxy-treated bioprostheses in various valve positions against the background of active infective endocarditis. Bulletin Bakulev Scientific Center of Cardiovascular Surgery RAMS 2009; 10: 22.

6.Lindblom D, Lindblom $U$, Qvist J, Lundstrom H. Longterm relative survival rates after heart valve replacement. J Am Coll Cardiol 1990: 15; 566-73.

7.Lung B, Garbarz E, Michaud P, Helou S, Farah B, Berdah $\mathrm{P}$, et al. Late results of percutaneous mitral commissurotomy in a series of 1024 patients: analysis of late clinical deterioration: frequency, anatomical findings, and predictive factors. Circulation 1999; 99 : 3272-8.

8.Lunga B, Baron G, Butchart EG, Delahae F, GohlkeBarwolf $C$, Lewang OW, et al. A prospective survey of patients with valvular heart disease in Europe: the Euro Heart Survey on Valvular Heart Disease. Eur Heart J 2003; 24: 1231-43.

9.Savage EB, Ferguson TB Jr, DiSesa VJ. Use of mitral valve repair: analysis of contemporary United States experience reported to the Society of Thoracic Surgeons National Cardiac Database. Ann Thorac Surg 2003; 75: 820-5.

10. Olofinskaya IE. The results of surgical treatment of acquired heart defects in elderly patients: risk factors, prognosis. Moscow 2009 ( dissertation Doctor of Medical Sciences).

11. Urmanbetov KS. Surgical treatment of acquired heart defects in elderly patients. Moscow 2015 (dissertation Doctor of Medical Sciences).

12.Schelbert EB, Vaughan-Sarrazin MS, Welk KF, Rosenthal GE. Hospital volume and selection of valve type in older patients undergoing aortic valve replacement surgery in the United States. Circulation 2005; 111:. 2178-82.

13. Roberts WC, Ko JM, Hamilton BAC. Comparison of valve structure, valve weight, and severity of the valve obstruction in 1849 patients having isolated aortic valve replacement for aortic valve stenosis (with or without associated aortic regurgitation) studied at 3 different medical centers in 2 different time periods. Circulation 2005; 112: 3919-29.

14.Roaldsen $\mathrm{MH}$. Demographic alteration in mitral valve surgery. Tromso 2006. (September 15, presentation).

15.Dotsenko NYa, Boev SS, Shekhunova IA. Heart disease in the elderly is the predominance of degenerative calcified stenosis of the aortic valve. Ther Ukrainian Med Bull 2008; 11: 35-50.

16. Order MHSD RF № 786n dated December 29, 2008 "On the procedure for the formation and approval of the state task for the provision in 2009 of high-tech medical care to citizens of the Russian Federation at the expense of federal budget appropriations.

17. Dell'Amore A, Aquino TM, Pagliaro M, Lamarra M, Zussa C. Aortic valve replacement with and without combined coronary bypass grafts in very elderly patients: early and long-term results. Eur J CardioThorac Surg 2012; 41: 491-8.

18.Revilla A, Lopez J, Vilacosta I, et al. Clinical and prognostic profile of patients with infective endocarditis who need urgent surgery. Eur Heart J 2007; 28: 65-71.

19.Guidelines on the management of valvular heart disease - version 2012. Available at: URL; /www.escardio.org/guidelines.

20.Maganti K, Rigolin VH, Sarano ME, et al. Valvular heart disease: diagnosis and management. Mayo Clinic Proc 2010; 85: 483-500.

21. Topilsky $Y$, Khanna AD, Oh JK, Nishimura RA, Enriquez-Sarano $M$, Jeon $Y B$., et al. Preoperative factors associated with adverse outcome after tricuspid valve replacement. Circulation 2011; 123: 1929-39. 
22.Detaint D, Sundt TM, Nkomo VT, et al. Surgical correction of mitral regurgitation in the elderly Outcomes and recent improvements. Circulation 2006; 114: 265-72.

23. Yoda $M$, Boethig $D$, Fritzsche $D$, Horstkotte $D$, Koerfer R, Minami K. Operative outcome of simultaneous carotid and valvular surgery. Ann Thorac Surg 2004; 78: 555-6.

24. Yoda $M$, Hata $M$, Sezai A, Minami K. Surgical outcome of simultaneous carotid and cardiac surgery. Surg Today 2011; 41: 67-71.

25. Kar S, Krishnaswamy A, Shishehbor MH, Cam A, Tuzcu EM, Bhatt DL, et al. Safety and efficacy of carotid stenting in individuals with concomitant severe carotid and aortic stenosis. Eurolntervention 2010; 6: 492-7.

26. Birchley D, Villaquiran J, Akowuah E, Lewis T, Ashley TS. Staged carotid endarterectomy under local anaesthetic in patients requiring cardiac surgery. Coll Surg Engl 2010; 92: 373-8.

27. Anselmi A, Gaudino M, Risalvato N, Lauria G, Glieca F. Asymptomatic carotid artery disease in valvular heart surgery: impact of systematic screening on surgical strategy and neurological outcome. Angiology 2012; 63: 171-7.

28.Stansby G, Macdonald S, Allisonet R, al. Asymptomatic carotid disease and cardiac surgery consensus. Angiology 2011; 62: 457-60. 